\title{
MONTE CARLO SIMULATION OF PARTIALLY ORDERED ATOMIC LAYERS ADSORBED ON THE MONOCRISTALLINE SURFACES
}

\author{
G. Wiatrowski ${ }^{a}$, J.C. Le Bossé ${ }^{b}$, J. LOPEz $^{b}$, J. RousseaU ${ }^{b}$ \\ AND I. ZASADA ${ }^{a}$ \\ a Department of Solid State Physics, University of Lódz \\ Pomorska 149/153, 90-236 Łódź, Poland \\ ${ }^{b}$ Laboratoire de Tribologie et de Dynamique des Systèmes \\ CNRS URA 855, Ecole Nationale d'Ingénieurs de St Etienne \\ 58 rue Jean Parot, 42023 St Etienne, France \\ CNRS URA 855, Ecole Centrale de Lyon, 69131 Ecully, France
}

\begin{abstract}
The calculations of the correlations functions and structure factors are performed by means of the Monte Carlo simulation. The results for $\mathrm{O} / \mathrm{Ni}(001)$ system are presented and compared with those obtained using self-consistent molecular field approximation and cumulant expansion method. The simulation for more complex system $\mathrm{CO} / \mathrm{Pt}(111)$ is also performed. The calculations of the topological correlations allow us to find the coverage dependence of the incoherent scattering amplitude which can be experimentally detected. PACS numbers: 68.35.-p
\end{abstract}

\section{Introduction}

\subsection{Oulline of the diffuse low-energy electron diffraclion problems}

For a couple of decades one of the most developed branches of research works is the surface science. According to new technologies and computer possibilities many phenomena are known much better now, however, some new problems have been arisen in this way. The problem of friction is one of the best examples [1,2]. Now, a new approach to this problem from the atomic point of view is a basis for the cooperation of mechanicians and physicists, although these two fields have been distinguished up to the moment.

6) The only way to understand complexity of the surface phenomena is to know point by point what is going on there, what are the real values of representative parameters, what kind of structures appears there and what kind of phenomena plays the most important role at contact points, in adsorbed layers and so on. The experiments using atomic force microscopes or dealing with diffuse low-energy electron diffraction (LEED) are the most sensitive between other techniques $[3,4]$. 
The latter has attracted our attention recently and some of the statistical aspects of diffuse LEED has been reported in the previous papers [5, 6]. Earlier, Le Bossé et al. [7] gave a general expression of the incoherent intensity of electrons backscattered at a single crystal surface partially covered with a disordered overlayer

$$
\begin{gathered}
I_{\text {incoh }}\left(k_{f}^{i} \leftarrow k_{i}^{+}\right)=\frac{4 \pi^{4} k}{A k_{i \perp}} \sum_{p=1}^{p_{\max }} \sum_{p^{\prime}=1}^{p_{\max }} \frac{k_{f \perp}^{2}}{\left|K_{f \perp}\right|^{2}} S\left(p, p^{\prime}, k_{f \|}-k_{i \|}\right) \\
\times \bar{K}_{1}\left(p, k_{f}^{-} \leftarrow k_{i}^{+}\right) K_{1}\left(p^{\prime}, k_{f}^{-} \leftarrow k_{i}^{+}\right),
\end{gathered}
$$

where

$$
S\left(p, p^{\prime}, k_{f \|}-k_{i \|}\right)=\sum_{j=1}^{N} \Gamma_{0 j}\left(p, p^{\prime}\right) \exp \left[-\mathrm{i}\left(k_{f \|}-k_{i \|}\right) T_{j}\right]
$$

is the Fourier transform of the site-occupancy pair correlation function $\Gamma_{0 j}\left(p, p^{\prime}\right)$ which is defined on the substrate surface lattice as follows:

$$
\Gamma_{0 j}\left(p, p^{\prime}\right)=\left\langle n_{0}(p) n_{j}\left(p^{\prime}\right)\right\rangle-\left\langle n_{0}(p)\right\rangle\left\langle n_{j}\left(p^{\prime}\right)\right\rangle .
$$

For other details see $[5,8,9]$. As usually the averages in (3) are performed over a grand canonical ensemble

$$
\langle A\rangle=\operatorname{tr}\{\rho A\}=\frac{\operatorname{tr}\{\exp [-\beta(H-\mu N) A]\}}{\operatorname{tr}\{\exp [-\beta(H-\mu N) A]\}}
$$

and a Hamiltonian $H$ of adsorbate-substrate and adsorbate-adsorbate interactions is following:

$$
H-\mu N=-\sum_{i=1}^{N}\left[E_{\mathrm{ads}}+\mu_{i}\right] n_{i}+\frac{1}{2} \sum_{i, j=1}^{N} U_{i j} n_{i} n_{j} .
$$

It is important to notice that the magnitude of pair interaction potential $U_{i j}$ in (5) as well as its sign is responsible for the crystalline structure of adsorbed layer even in the nearest-neighbour approximation. It has been shown using different approaches described below [10]. For $\mathrm{O} / \mathrm{Ni}(001)$ system with strong repulsive interactions and coverages $\theta<0.25$ it is only possible to obtain $\mathrm{c}(2 \times 2)$ superstructure instead of $p(2 \times 2)$ one. The latter case can be generated using next nearest and farther neighbour approximation in Monte Carlo simulation what is described in Sec. 2.

\subsection{Theoretical approach to slatistical aspects of diffuse LEED problem}

Up to now, site-site correlation functions (3) have been calculated using two approximated methods [10]: the self-consistent molecular field approximation (SCMFA) and cumulant expansion method (CEM). Then, it is possible to determine structure factors (2) in some restricted cases: low coverage limit or weak interaction potentials in (5). The SCMFA method gives the following approximated expressions for the coverage $\theta$ and for the infinite number of correlation functions $\Gamma_{m n}$ :

$$
\theta=\frac{1}{1+\exp \left[\beta\left(-E_{\mathrm{ads}}-\mu+4 U^{*} \theta\right)\right]},
$$




$$
\begin{aligned}
& {\left[1-\beta \theta(1-\theta) U^{*}\right] \Gamma_{10}+\beta \theta(1-\theta) U^{*}\left(2 \Gamma_{11}+\Gamma_{20}\right)=-\beta \theta^{2}(1-\theta)^{2} U^{*},} \\
& \Gamma_{m n}+\beta \theta(1-\theta) U^{*}\left(\Gamma_{m-1 n}+\Gamma_{m+1 n}+\Gamma_{m n-1}+\Gamma_{m n+1}\right)=0,
\end{aligned}
$$
where $U^{*}$ is the renormalized interaction potential, $\theta$ is surface coverage and $\beta=\left(k_{\mathrm{B}} T\right)^{-1}$.

The second approach (CEM) at the first stage using Dirac's $\delta$-funclion method [11] gives the exact expressions for mean occupation $\left\langle n_{1}\right\rangle$ and for "pure" correlation function $\left\langle n_{1} n_{j}\right\rangle$ [12]

$$
\begin{aligned}
& \left\langle n_{1}\right\rangle=A_{1}+A_{2} \sum_{i}\left\langle n_{i}\right\rangle+A_{3} \sum_{i, j}^{\prime}\left\langle n_{i} n_{j}\right\rangle+A_{4} \sum_{i, j, k}^{\prime}\left\langle n_{i} n_{j} n_{k}\right\rangle+A_{5}\left\langle n_{2} n_{3} n_{4} n_{5}\right\rangle, \\
& \left\langle n_{1} n_{m}\right\rangle=A_{1}\left\langle n_{m}\right\rangle+A_{2} \sum_{i}\left\langle n_{i} n_{m}\right\rangle+A_{3} \sum_{i, j}^{\prime}\left\langle n_{i} n_{j} n_{m}\right\rangle \\
& \quad+A_{4} \sum_{i, j, k}^{\prime}\left\langle n_{i} n_{j} n_{k} n_{m}\right\rangle+A_{5}\left\langle n_{2} n_{3} n_{4} n_{5} n_{m}\right\rangle,
\end{aligned}
$$

where coefficients $A_{k}$ are the known functions of temperature parameter $\beta$ and interaction potential $U$ for s.q. substrate structure in the nearest neighbour approximation. Then, using the following cumulant expansion for multi-correlations in (10):

$$
\begin{aligned}
& \left\langle n_{1}\right\rangle=\theta \\
& \left\langle n_{1} n_{2}\right\rangle=\Gamma_{12}+\theta^{2}, \\
& \left\langle n_{1} n_{2} n_{3}\right\rangle=\left\{n_{1} n_{2} n_{3}\right\}+\theta\left(\Gamma_{12}+\Gamma_{13}+\Gamma_{23}\right)+\theta^{3} \\
& \left\langle n_{1} n_{2} n_{3} n_{4}\right\rangle=\left\{n_{1} n_{2} n_{3} n_{4}\right\}+\left(\Gamma_{12} \Gamma_{24}+\Gamma_{13} \Gamma_{24}+\Gamma_{14} \Gamma_{23}\right) \\
& \quad+\theta\left(\left\{n_{1} n_{2} n_{3}\right\}+\left\{n_{1} n_{2} n_{4}\right\}+\left\{n_{1} n_{3} n_{4}\right\}+\left\{n_{2} n_{3} n_{4}\right\}\right) \\
& \quad+\theta^{2}\left(\Gamma_{12}+\Gamma_{13}+\Gamma_{14}+\Gamma_{23}+\Gamma_{24}+\Gamma_{34}\right)+\theta^{4}
\end{aligned}
$$

and neglecting cumulants $\left\{n_{1} n_{2} \ldots n_{k}\right\}$ of the order $k$ higher than 2 the systcm (9)-(10) is transformed into the approximated expressions for the coverage $\theta$ and correlation functions $\Gamma_{m n}$ [13]

$$
\begin{aligned}
& \theta=A_{1}+4 A_{2} \theta+6 A_{3} \theta^{2}+4 A_{4} \theta^{3}+A_{5} \theta^{4}+\left(2 A_{3}+4 A_{4} \theta\right)\left(2 \Gamma_{11}+\Gamma_{20}\right) \\
& \quad \quad+a_{5}\left(2 \Gamma_{11}^{2}+\Gamma_{20}^{2}\right), \\
& \Gamma_{10}+\theta(1-\theta) K_{1}(\theta)\left(2 \Gamma_{11}+\Gamma_{20}\right)=H_{1}(\theta), \\
& \Gamma_{m n}+K_{1}(\theta)\left(\Gamma_{m-1 n}+\Gamma_{m+1 n}+\Gamma_{m n-1}+\Gamma_{m n+1}\right)=0, \\
& H_{1}(\theta)=A_{2}+A_{3}(1+\theta)+A_{4} \theta(3-2 \theta)+2 A_{5} \theta^{2}(1-\theta), \\
& K_{1}(\theta)=-\left[A_{2}+3 A_{3} \theta+3 A_{4} \theta^{2}+A_{5} \theta^{3}\right] .
\end{aligned}
$$

These two methods give the reliable results only for very low coverages and week interactions between adsorbates in nearest neighbour approximation. To extend investigations of diffuse LEED statistical aspects for arbitrary parameter region and more complicated systems we are obliged to perform the simplest Monte Carlo simulation of adlayer. 


\section{Monte Carlo simulation of adsorbed layer $\mathrm{O} / \mathrm{Ni}(001)$ and $\mathrm{CO} / \mathrm{Pt}(111)$}

The object of this Monte Carlo simulation is to provide estimation of the statistical mechanical Boltzmann averages of site-site pair occupation correlation functions (3) of the adsorbed gas atoms or molecules onto certain substrate surface as $\mathrm{O} / \mathrm{Ni}(001)$ or $\mathrm{CO} / \mathrm{Pt}(111)$. To obtain a Markov chain of representative configurations $C_{t}$ with energy $E_{t}$ we perform a biased random walk in the phase space called the importance sampling [14]. The transition probabilities for any local change of single adatom position is determined as usually in the following way:

$$
p_{i \rightarrow i+1}=\left\{\begin{array}{ccc}
\exp \left(-\beta\left|E_{i+1}-E_{i}\right|\right) & \text { for } & E_{i+1}>E_{i}, \\
1 & \text { for } & E_{i+1} \leq E_{i} .
\end{array}\right.
$$

For any configuration $C_{t}$ which consists of many local adatom changes (practically it is a number of adatoms taken into consideration) it is easy to calculate temporary mean value, namely

$$
\bar{\Gamma}_{m n}(t)=\frac{1}{\text { TOTAL }} \sum \Gamma_{m n}(t)
$$

where the summation is performed over all simultaneously occupied adatom posi-
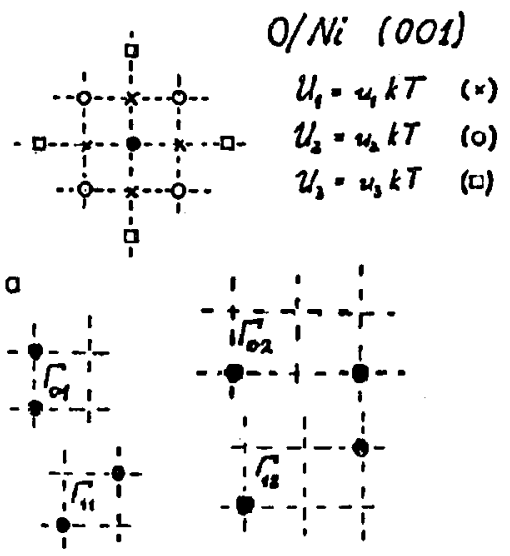

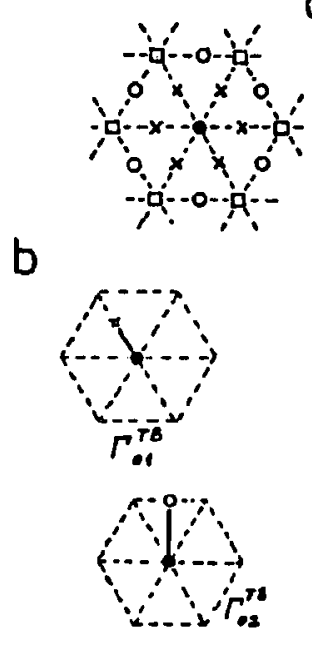

b

$\operatorname{Col} P t(111)$
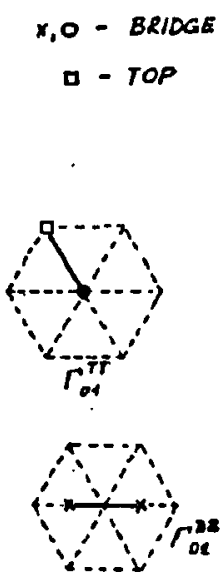

Fig. 1. Suggested model for simulation of statistical distribution of (a) oxygen adatoms on $\mathrm{Ni}(001)$ substrate; (b) carbon-oxide admolecules on $\mathrm{Pt}(111)$ substrate with different interaction energies $u_{1}, u_{2}$ and $u_{3}$. Below pairs of correlated adatoms are shown contributing to (a) $\Gamma_{i j}$ and (b) $\Gamma_{i j}^{r, s}(r, s=$ TOP, BRIDGE) pair correlation functions (32). 
tions of a given type $(m n)$ - see Fig. 1. TOTAL denotes the number of all possible pairs $(m n)$ (occupied or not) on the adlayer in question. Hence, we obtain mean value of parameter $\Gamma_{m n}(t)$ along the Markov chain of the lenght $L$

$$
\Gamma_{m n} \equiv\left\langle\bar{\Gamma}_{m n}\right\rangle_{\mathrm{M}-\mathrm{C}}=\frac{1}{L} \sum_{t=N_{0}}^{N_{0}+L}\left\langle\bar{\Gamma}_{m n}(t)\right\rangle .
$$

In (22) the summation is started with the configuration $C_{N_{0}}$ which is chosen in such a way that the contribution of initial configuration $C_{0}$ in the ensemble is finally lost. This configuration is generated by adding adatoms onto the substrate sites chosen randomly but its final occupation is according to probability (20) which can be written in the following form to shorten this procedure:

$$
P_{+} \propto \exp \left(-\Delta n_{1} u_{1}-\Delta n_{2} u_{2}+\Delta n_{3} u_{3}\right)
$$

where $\Delta n_{i}$ is a number of created $i$-bonds with energy $U_{i}=u_{i} k_{\mathrm{B}} T, i=1,2,3$, respectively (compare Fig. 1).

As usually dealing with a pseudo-random number generator we should be very careful to get right results especially close to the critical regions where the systems become very sensitive and unstable [15] and correlation length can be comparable with the linear size $N$ of the simulated mesh. For this reason the number of different characteristics is checked, namely "time" variation of particular Markov processes in Fig. 2 or their distances $L^{2}(t)$ for too small mesh compared with the biggest piece of 160000 sites (Fig. 3).

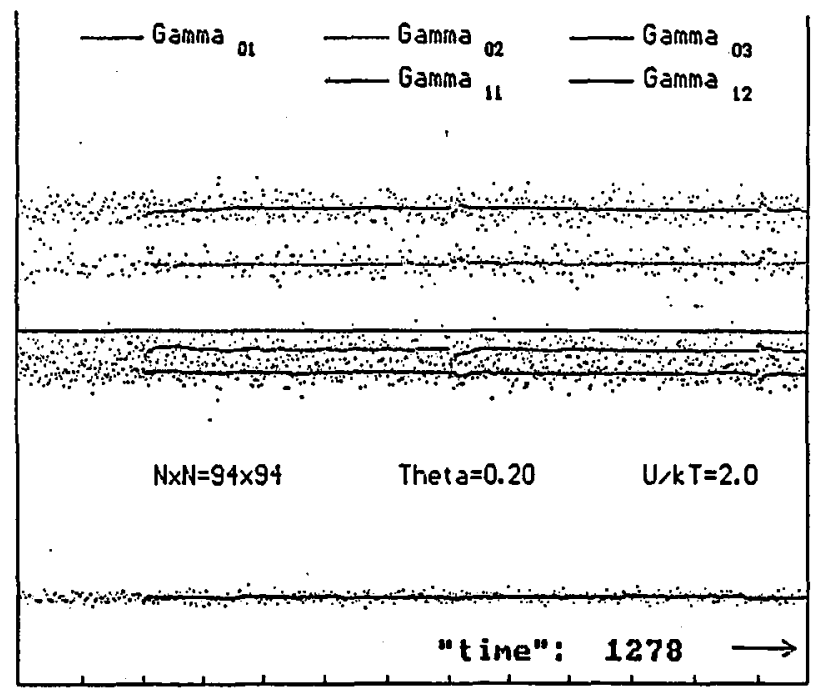

Fig. 2. Artificial time evolution of certain Markov process for $\mathrm{O} / \mathrm{Ni}(001)$ system with n.n. approximation and $u_{1}=2.0 ; \theta=0.2$. Drops denole temporary values of respective correlation functions (33), solid lines represent mean values recalculated every $t=500$ configurations to get coarse grained type averaging with $N_{0}=200$. 


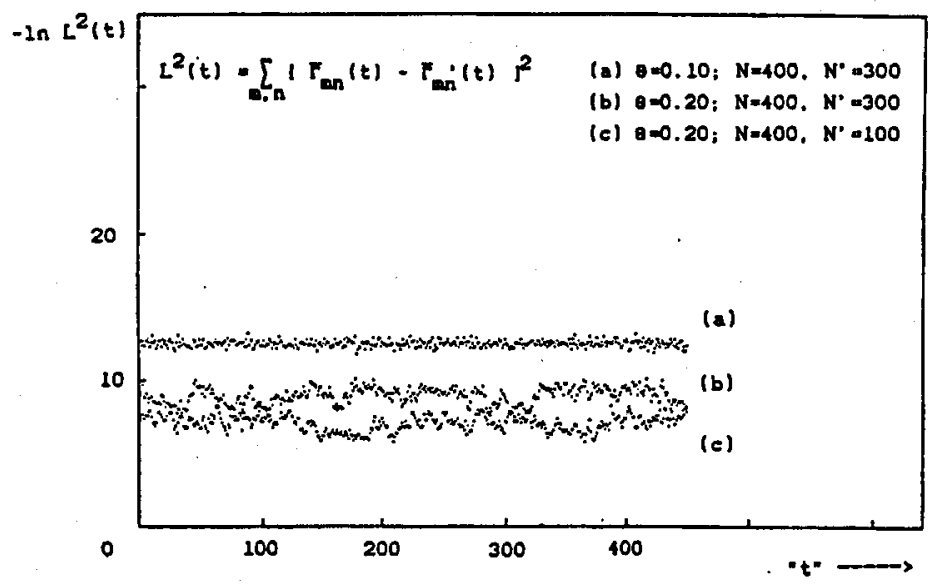

Fig. 3. Distance $L^{2}(t)$ of different Markov chains vs. "lime" for different sizes $N^{\prime}$ compared with the biggest simulated piece $N \times N=400 \times 400$ when $\theta=0.10$ and 0.20 , respectively.

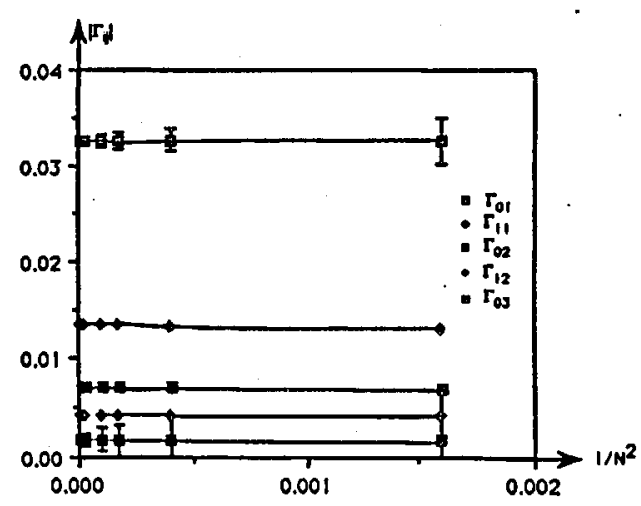

Fig. 4. Pair correlation function $\Gamma_{i j}$ of adsorbed partially ordered layer $\mathrm{O} / \mathrm{Ni}(001)$ for $\theta=0.20$ and $u_{1}=2.0$ (n.n. approx.) with periodic boundary conditions plotted vs. inverse square dimension of the lattice. Left hand side results show the good tendency to thermodynamic limit for $N \longrightarrow \infty$ and $\theta=$ const.

In order to omit the final size problems we perform a linear extrapolation of the correlation functions $\Gamma_{i j}$ versus $N^{-2}$ for $N \longrightarrow \infty$. It is worth noticing that for usual diffuse LEED parameters, outside of the critical regions, this procedure is not needed, see Fig. 4.

As it was told before, a sufficient number of local changes with probability (20) is performed to close any configuration $C_{t}$, therefore there is no problem with correlation of successive configurations. However, it should be verified carefully whether the slowing down close to the critical region $\theta \rightarrow 0_{\text {crit }}$ allows one to ob- 
tain reliable results after reliable time of simulations. For that reason the different lengths $L$ of the representative Markov chain are considered together with the averaging procedure of coarse grained type (22), where the mean values for different parts of Markov process (Fig. 2) are compared. Then, if they are reasonable stable over an extended number of coarse grained a verages we consider this value to be the ensemble average of the correlation function in question.

It should be noticed that the presented simulation is performed not to generate realistic physical adsorption-desorption process observed during the LEED experiment. In order to describe the statistical distribution of adatoms at the substrate surface in question we can perform averaging procedure (22) dealing only with the representative subset of canonical ensemble of configurations $\left\{C_{t}\right\}_{\text {can }}$.

\section{Conclusions and final remarks}

Two theoretical methods SCMFA and CEM reviewed above give the similar infinite set of linear algebraic equations for which solutions $\Gamma_{m n}$ are close to those obtained by Monte Carlo simulations only for a certain restricted area: (1) if potential interaction is rather weak compared with $k T, 0<\theta<\theta_{\text {cirt }}$; (2) for any potential interaction but in the low coverage limit $\theta<0.05$ (see Fig. 5 and 6 ).

The closer to $\theta_{\text {crit }}$ the greater number of bigger islands of well-defined superstructures occurs, therefore the only Monte Carlo simulation results are correct (see Fig. 7a-d).

It is worth noticing that the more the system is free (for small coverage) the more configurations could be achieved (the smaller determination of successive M-C configurations $C_{t}$ ) and it means the bigger fluctuations of the resulting parameters $\Gamma_{m n}(t)$ - compare the plot for $\theta=0.1$ with the rest of the results in Fig. 6.

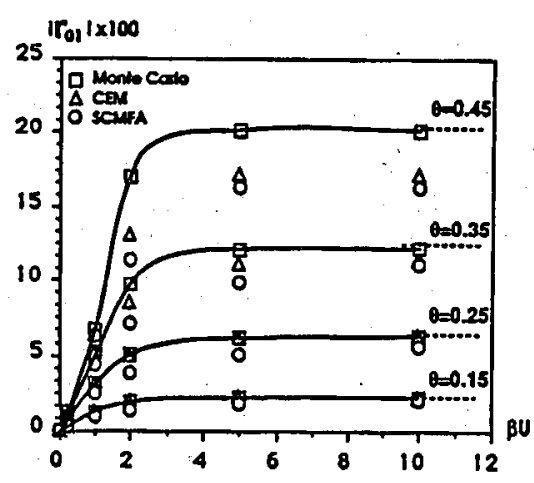

Fig. 5. The strongest pair correlation function $\Gamma_{01}$ plotted vs. interaction energy parameter $\beta U$ for different coverages $\theta=0.15,0.25,0.35$ and 0.45 according to three presented methods. The dash line represents the asymptotic value of $\theta^{2}$. 
$\Gamma_{\text {ij }}$

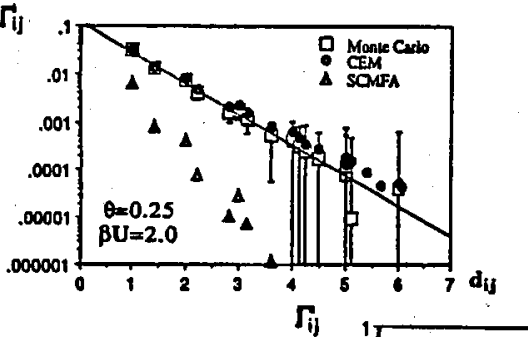

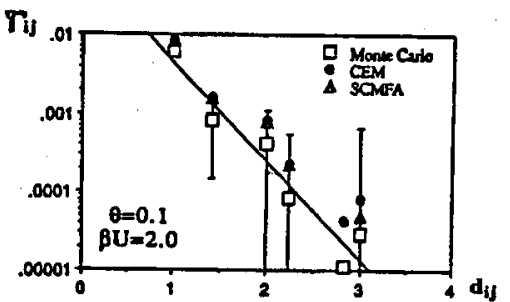

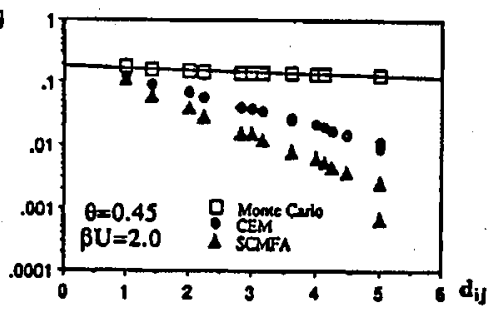

Fig. 6. Pair correlation function $\Gamma_{i j}$ vs. distance of correlated adatoms $d_{i j}$ for repulsive interaction of adjacent sites $u_{1}=2.0$ and various coverages $\theta=0.1,0.25$ and 0.45 . Solid line represents the exponential decay of correlations with a distance.

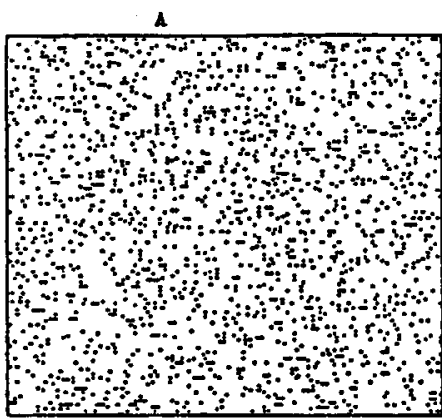

c.

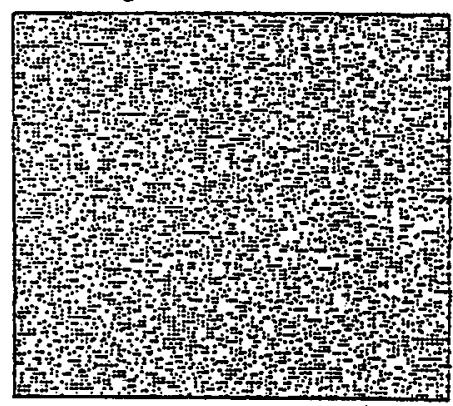

B

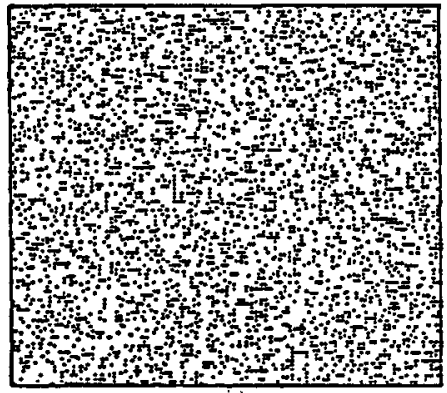

D

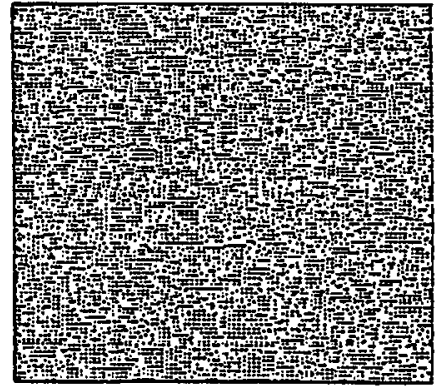

Fig. 7. Snapshot pictures of a system $\mathrm{O} / \mathrm{Ni}(001)$ with interactions $u_{1}=10.0, u_{2}=2.5$ and $u_{3}=-0.5$ for $N \times N=179 \times 179$ and different coverages (a) $\theta=0.05$, (b) $\theta=0.10$, (c) $\theta=0.15$ and (d) $\theta=0.20$. 
Finally, increasing the coverage parameter $\theta$ from the diffuse LEED region to the classical one we can observe the contribution of the structure factor of the system to the LEED pattern, especially just below the critical region when $\mathrm{p}(2 \times 2)$ superstructure islands start to grow up. This feature is shown in Fig. 8 where for successive coverages $\theta$ in the case of $\mathrm{O} / \mathrm{Ni}(001)$ system one can observe the presence of sharp structures near fractional order peaks which is essential for the difluse LEED experiment.
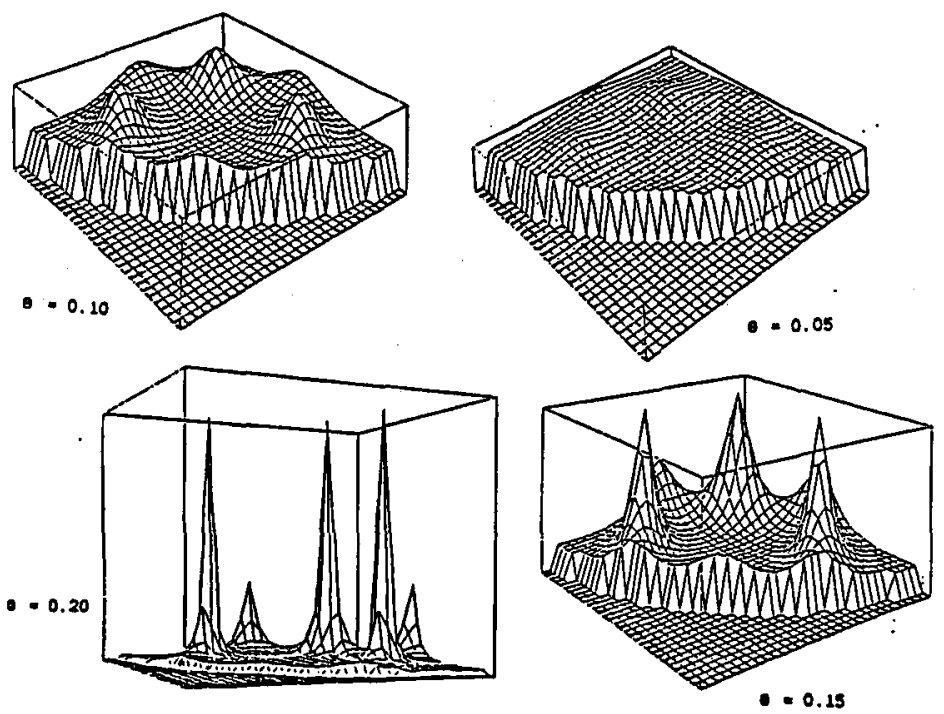

Fig. 8. Perspective representation of the structure factor $S(q)$ for the system $\mathrm{O} / \mathrm{Ni}(001)$ as in Fig. $7 ; U_{1}=10 k_{\mathrm{B}} T, U_{2}=2.5 k_{\mathrm{B}} T, U_{3}=-0.5 k_{\mathrm{B}} T$.

From the other hand, the diffuse LEED intensity obtained via the structure factor $S(q)$ for the case of more than one kind of chemisorption sites like $\mathrm{CO} / \mathrm{Pt}(111)$ system [6] is not tractable with the use of the $Y$ function method contrary to the suggestion of Saldin et al. [16]. The extension of our previous simulations to the second case of $\mathrm{CO} / \mathrm{Pt}(111)$-system (see Fig. 1b) allowed us to obtain the snapshots of adsorbed carbon-monooxide molecules. Because we have obtained the proper superstructure islands known from other experiments, so we can conclude that the comparison experience/theory should take into consideration the contribution of structure factor $S(q)$. The final proof is the task for the next paper in progress as the continuation of the just presented results for $\mathrm{CO} / \mathrm{Pt}(111)$ simulation.

\section{References}

[1] D. Dowson, J. Lubr. Technol. Trans. ASME 99, 383 (1978).

[2] M. Hirano, K. Shinjo, Phys. Rev. B 41, 11837 (1990).

[3] C.M. Mate, G.M. McClelland, R. Erlandsson, S. Chiang, Phys. Rev. Lett. 59, 1942 (1987). 
[4] G.S. Blackman, M.L. Xu, D.F. Ogletree, M.A. Van Hove, G.A. Somorjai, Phys. Rev. Lelt. 61, 2352 (1988):

[5] J.C. Le Bossé, J. Lopez, J. Rousseau, I. Zasada, L. Wojtczak, J. Phys., Condens. Maller 2, 3143 (1990).

[6] J.C. Le Bossé, J. Lopez, J. Rouseau, I. Zasada, J. Phys., Condens. Maller 4, 1671 (1992); 4, 1685 (1992).

[7] J.C. Le Bossé, J. Lopez, J. Rousseau, I. Zasada, J. Phys. C, Solid Stale Phys. 21, 565 (1988).

[8] L. Wojtczak, I. Zasada, J.C. Le Bossé, J.Lopez, J. Rousseau, Acta Physicae Superficierum I, 3 (1990).

[9] L. Wojtczak, I. Zasada, J.C. Le Bossé, J. Lopez, J. Rousseau, Acta Phys. Pol. A 80, 631 (1991).

[10] G. Wiatrowski, J.C. Le Bossé, J. Lopez, I. Zasada, Surf. Sci. 265, 229 (1992).

[11] G. Wiatrowski, J. Mielnicki, T. Balcerzak, A. Urbaniak-Kucharczyk, J. Magn. Magn. Mater. 73, 89 (1988).

[12] N. Matsudaira, J. Phys. Soc. Jpn. 35, 1593 (1973).

[13] J. Mielnicki, T. Balcerzak, G. Wiatrowski, Phys. Status Solidi B 161, 335 (1990).

[14] L.D. Fosdick, in: Monle Carlo Computation on the Ising Lallice, in series Methods in Computational Physics, Eds. B. Adler, S. Fernbach, M. Rotenberg, Vol. 1, Academic Press, New York 1963, p. 245.

[15] K. Binder, Monte Carlo Method in Statistical Physics, Springer-Verlag, Berlin, Heildelberg, New York 1979, p.10.

[16] D.K. Saldin, J.B. Pendry, M.A. Van Hove, G.A. Somorjai, Phys. Rev. B 31, 1216 (1985). 